\title{
Commentary on "Long-term outcomes of Graves' disease in children and adolescents receiving antithyroid drugs"
}

\author{
Minsun Kim \\ Department of Pediatrics, Jeonbuk \\ National University Medical School, \\ Research Institute of Clinical Medicine \\ of Jeonbuk National University- \\ Biomedical Research Institute of \\ Jeonbuk National University Hospital, \\ Jeonju, Korea
}

This article ${ }^{1)}$ investigates long-term progression of Graves' disease (GD) patients who were followed up for $>5$ years. GD is the most common cause of hyperthyroidism in children, accounting for about 95\% of cases in Korea. ${ }^{2)}$ The optimal GD treatment in children is controversial. Antithyroid drugs (ATDs) are convenient and prevent permanent hypothyroidism and are primarily used as an initial treatment for children. ${ }^{3)}$ However, the rate of GD remission with ATD treatment alone is low, and there are no clinical guidelines on the timing of ATD treatment cessation in children and adolescents. ${ }^{1)}$ Additionally, there are few studies about the efficacy and disease progression in children and adolescents on long-term ATD therapy. ${ }^{1,4)}$

Relapse and remission rates after discontinuing ATD therapy vary by study. Factors associated with remission include higher body mass index standard deviation score, smaller goiter size, lower initial T3 level, and older age. ${ }^{5)}$ It also has been proposed that higher antimicrosomal antibody level, higher thyroid-stimulating hormone (TSH) level, and lower antithyroglobulin antibody level are related to remission, ${ }^{5)}$ although any one of these factors alone is insufficient to predict remission of hyperthyroidism. ${ }^{1,4)}$

This study demonstrated the value of TSH-binding inhibitory immunoglobulin (TBII) as a predictor of remission during the 5 years after ATD treatment. They showed that the remission group had significant relationships with a shorter time to TBII normalization and lower level of TBII. Although this article has some limitations, it is a relatively long-term analysis of patient data over 5 years, and it will be a valuable resource for researchers who want to predict the course of GD patients in children and adolescents through larger samples and longer-term data. It will also serve as a cornerstone for long-term treatment guidelines.

\section{Conflict of interest}

No potential conflict of interest relevant to this article was reported.

\section{References}

See the article"Long-term outcomes of Graves' disease in children and adolescents receiving antithyroid drugs" via https://doi.org/10.6065/ apem.2040286.143.

Address for correspondence: Minsun Kim

Department of Pediatrics, Jeonbuk National University Hospital, 20 Baekje-daero, Deokjin-gu, Jeonju 54907, Korea

Email: children@jbnu.ac.kr

https://orcid.org/0000-0002-36177823
1. Rho JG, Kum CD, Seo YJ, Shim YS, Lee HS, Hwang JS. Long-term outcomes of Graves' disease in children and adolescents receiving antithyroid drugs. Ann Pediatr Endocrinol Metab 2021;26:266-71.

2. Song SM, Youn JS, Ko JM, Cheon CK, Choi JH, Yoo HW. The natural history and 2010;53:585-91.

3. Roh JG, Park KJ, Lee HS, Hwang JS. Thyrotoxic hypokalemic periodic paralysis due to Graves' disease in 2 adolescents. Ann Pediatr Endocrinol Metab. 2019;24:133-6.

4. Azizi F. Long-term treatment of hyperthyroidism with antithyroid drugs: 35 years of personal clinical experience. Thyroid 2020;30:1451-7.

5. Glaser N, Styne D. Organization of pediatric endocrinologists of Northern California Collaborative Graves' Disease Study Group. Predicting the likelihood of remission in children with Graves' disease: a prospective, multicenter study. Pediatrics 2008;121:e481-8. prognostic factors of Graves' disease in Korean children and adolescents. Korean J Pediatr 